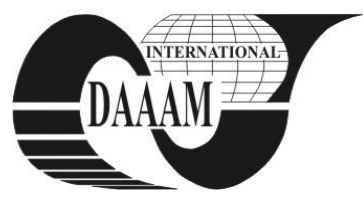

\title{
THE SUPERFICIAL HARDENING OF INNER CILINDRICAL SURFACES
}

\author{
LEUCA, T[eodor]; NAGY, S[tefan]; MICH - VANCEA, C[laudiu] \& NAGY, A[drian]
}

\begin{abstract}
This paper deals with the numerical computation of the quasi-stationary electromagnetic field within ferromagnetic pipes in an industrial inductor. An accurate computation of the electric and magnetic field inside the supposed part is done by the mean of finite element method FEM. Eddy current density and power losses are evaluated as a function of amplitude and frequency of the exciting current. The knowledge of eddy currents and power losses allows the estimation of optimal supply frequency. The software allows, beside the structure and rotation symmetry, the interactive development of a mesh that could have any shape.
\end{abstract}

Key words: hardening, induction heating, eddy currents, numerical modeling, electromagnetic field

\section{INTRODUCTION}

Numerical analyses of phenomena that occur in the walls of metal pipes are needed to verify the characteristics of the materials and to simulate the behavior for different actions, minimum resistance dimensions, etc., (Behulova, 2009). The paper proposes a method for numerical analysis of the electromagnetic field when processing the pipes using the induction heating.

We intend to establish a complex system to simulate the whole process of induction heating - to be able to use it as an active aid in the design optimization of the heating equipment. The first step in the analysis is the solution of the electromagnetic field problem - performed by means of the finite element method (Hantila, 2001) \& (Fluerasu, 2008). Contribution on optimal design of induction heating equipment, electric equipment choice in technological installations and computation of the energetic parameters are presented in (Arion, 2008). Simulations have been made using a professional CAD software package for electromagnetic analysis.

In the following an outline of the field problem will be presented followed by the description of the numeric approach. Results of simulations are then presented for a configuration in which superficial hardening was done on a metallic, linearly magnetic sample (interior heating). The influence of mesh coarseness on the result is pointed out. A future work will integrate a thermal analysis module in the system.

The mathematic model for the electromagnetic field is present in (Hantila, 2001).

Usually a potential solution to the problem is preferred. In the following we refer to the A, V- $\psi$ one (Hantila, 2001). This means using magnetic vector potential in $\Omega_{\mathrm{c}}$ together with scalar electric potential combined with the use of magnetic scalar potential in the non-conducting area (Ciric, 2007).

In $\Omega_{\mathrm{c}}$ the use of potential $\mathbf{A}$ and $\mathrm{V}$ yields the following second order equation:

$$
\nabla \times \vartheta \nabla \times \mathbf{A}+\sigma \frac{\partial \mathbf{A}}{\partial t}+\sigma \nabla V=0
$$

which includes also the divergence free condition verified by the induced eddy current $\mathbf{J}\left(\boldsymbol{\vartheta}=\boldsymbol{\mu}^{-\mathbf{1}}\right)$. To obtain a sighificant reduction of computing effort in $\Omega_{0}$ we use the following decomposition:

$$
\mathbf{H}=\mathbf{H}_{0}-\nabla \phi
$$

where $\mathbf{H}_{0}$ is the field due to source currents and can be easily computed by Biot-Savart-Laplace relation, and $\phi$ is reduced magnetic scalar potential.

If conductivity is constant in domain $\Omega_{\mathrm{c}}$ it can be shown (Arion 2008) that scalar potential V is constant in $\Omega_{\mathrm{c}}$ and hence it can be chosen to be zero. Hence A becomes in fact a modified vector potential. For the numeric solution of the problem an implementation of the finite element method have been used. The nodal elements are employed and the interpolations are the following:

Denote by $\partial \Omega_{\mathrm{c}}$ the surface bordering the conducting material and by $\partial \Omega_{0}$ the outer boundary. In the free space region $\Omega_{0}$ the following Maxwell equations are valid in the quasi-stationary limit (Hantila, 2001).

where:

$$
\begin{aligned}
\mathbf{A} & \approx \sum_{k} A_{k} \mathbf{N}_{k} \\
\mathrm{~V} & \approx \sum_{k} \mathrm{~V}_{j} N_{j} \\
\psi & \approx \sum_{\mathrm{j}} \psi_{j} N_{j}
\end{aligned}
$$

$$
\mathbf{N}_{k}=N_{j} \mathbf{e}_{s}, \quad \mathrm{~s}=\mathrm{x}, \mathrm{y}, \mathrm{z}
$$

and $\mathrm{e}_{\mathrm{x}}, \mathrm{e}_{\mathrm{y}}$ and $\mathrm{e}_{\mathrm{z}}$ are the Cartesian unit vectors. $N_{\mathrm{j}}$ are the shape functions associated with node $j$ - the usual roof functions.

Upon application of Galerkin technique the following formulation is obtained:

$$
\begin{gathered}
\int_{\Omega_{c}} N_{i}\left(\nabla \times \vartheta \nabla \times A-\nabla \vartheta \nabla \cdot A+\sigma \frac{\partial A}{\partial t}+\nabla V\right) d \Omega=0 \\
\int_{\Omega_{C}} N_{i}\left[\nabla \cdot\left(-\sigma \frac{\partial A}{\partial t}-\nabla V\right)\right] d \Omega=0 \\
\int_{\Omega_{0}} N_{i} \nabla \cdot \mu \nabla \psi d \Omega=0
\end{gathered}
$$

The usual integration by parts technique yields the weak formulation - and following the introduction of approximations (3) into the weak formed obtained from (4) the algebraic system of equations is obtained.

\section{RESULTS}

In the following figure we present a configuration used in superficial hardening treatments. The sample has the following characteristics: internal radius $30[\mathrm{~mm}]$, external radius 35 [mm] and length 200 [mm].

The induction coil, placed in interior position (as can be seen in Fig. 1 has a rectangular cross section. The opening of the coil is $320^{\circ}$. The air gap between the pipe and the inductor is increased from $1.5[\mathrm{~mm}]$ to $2.5[\mathrm{~mm}]$ in order to establish the influence of the air gap on the induction heating process. The amplitude of the current density flowing through the coil is 61 $\mathrm{A} / \mathrm{mm}^{2}$. The coil positions for which we have computed the results are presented in Fig. 1. 


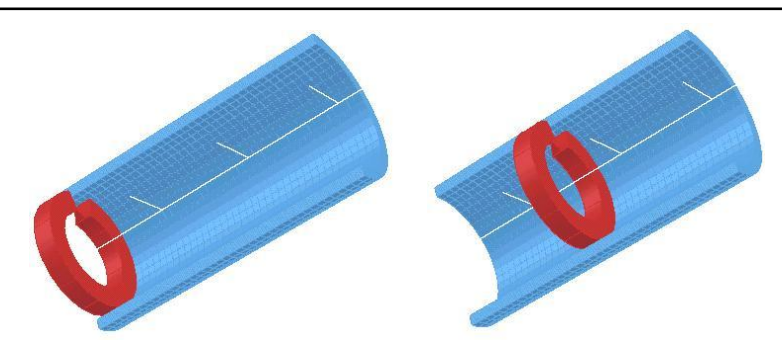

Fig. 1. Geometry considered for the case of superficial hardening (first position and second position)

First we started by establishing the influence of the air gap upon the induced eddy currents in the pipe for both cases. Results of these simulations are presented in Figures 2 and 3.

Figure 4 and 5 represent results of the simulations made in order to point out the influence of the air gap on the simulation values of the induced Joule losses.

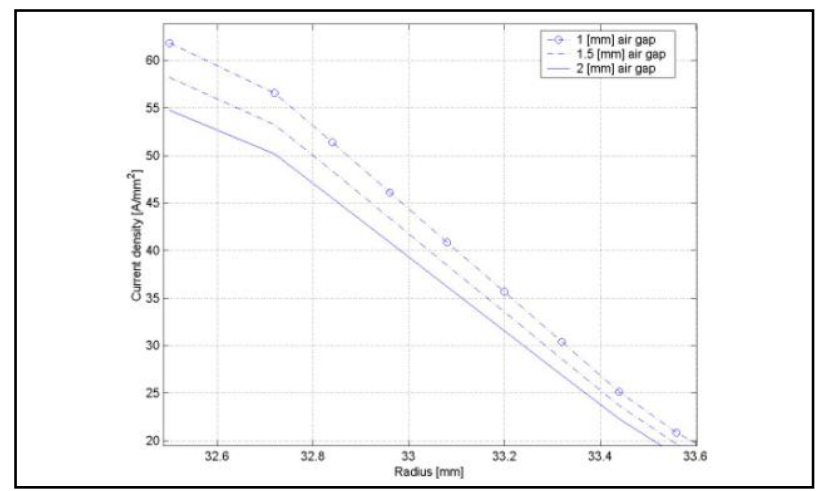

Fig. 2. The influence of air gap on the eddy currents induced in the inner cylindrical surface, frequency $f=8[\mathrm{kHz}]$. First position of the inductor

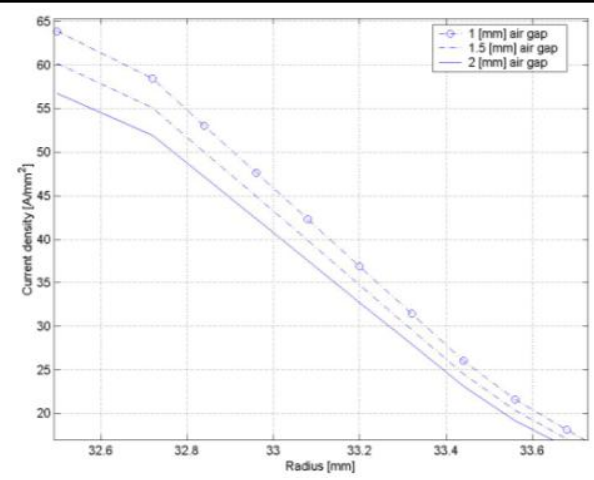

Fig. 3. The influence of air gap on the eddy currents induced in the inner cylindrical surface, frequency $\mathrm{f}=8[\mathrm{kHz}]$. Second position of the inductor

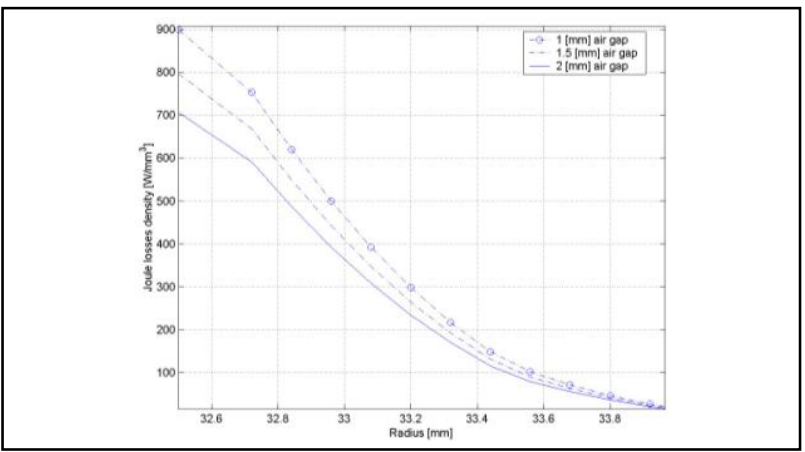

Fig. 4. The influence of air gap on the joule losses induced in the inner cylindrical surface, frequency $\mathrm{f}=8[\mathrm{kHz}]$. First position of the inductor

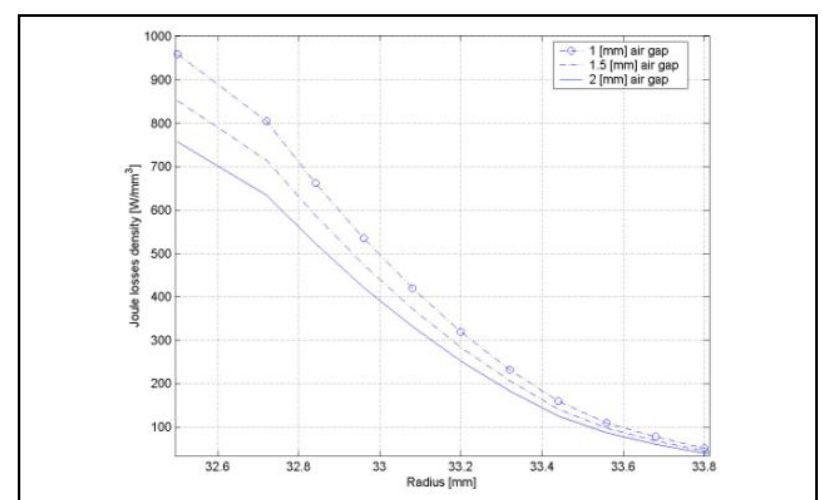

Fig. 5. The influence of air gap on the joule losses induced in the inner cylindrical surface, frequency $f=8[\mathrm{kHz}]$. Second position of the inductor

\section{CONCLUSIONS}

The space distribution of the field values offers a more thorough analysis of the complex induction heating process. The analysis of the dependencies offers important information on correlating the technological requirements with the electrical parameters of the heating equipment.

The precise knowledge of the distribution of eddy currents and power density allows the analysis and the optimal design of the electromagnetic equipment. Because this information is not available through regular analytic methods we have to use modern mathematical methods allowing the solving of complex electromagnetic field problem, having thousands of unknown values in discrete representation.

The numerical analysis of the electromagnetic field is a major advantage in studying the inductive heating processes which resulted in providing a uniform electromagnetic field for the work pieces. An important aspect treated in the paper is the influence of the electromagnetic field source's parameters, such as frequency and position of the field source. The effects of induced eddy currents in the walls of pipes will be the next steps for researches in this field.

\section{REFERENCES}

Arion, M.; Leuca, T., Cheregi, G.R., Soproni, V., \& Hathazi F.I., (2008). Numerical analysis of the coupled electromagnetic and thermal field question within industrial induction heating systems, Simpozionul National de Electrotehnică Teoretică, 5-7 Iulie 2008, Bucuresti , ISBN 978-606-521-045-5, pp. 79-84

Behulova, M. (2009). NumericalAnalysis of the Cooling of Thin-Walled Pipes from a TRIP Steel, Annals of DAAAM for 2009 \&Proceedings of the 20th International DAAAM Symposium, 25-28th November 2009, Vienna, Austria, ISSN 1726-9679, ISBN 978-3-901509-70-4, Katalinic, B. (Ed.), pp. 1015-1016

Fluerasu, C.; Fluerasu, Corina, (2008). Calcul des regimes transitoires thermiques dans des materiaux avec proprietes dependantes de la temperature, Revue Roumaine des Sciences Techniques Serie Electrotechnique et Energetique, Vol. 53, No. 3, pp. 269-278, ISSN: 0035-4066

Hantila, F.; et all (2001). The numeric computation of eddy currents, Ed. ICPE, ISBN 973 - 8067 - 31 - 6, Bucharest, (in Romanian), ISBN 973-98322-0-2

Ciric, I.; Hantila, F. (2007). An Efficient Harmonic Method for Solving Nonlinear Time-Periodic Eddy-Current Problems, IEEE Transaction on Magnetics, no. 4, vol. 43, pp. 11851188 